\title{
si-MALAT1 attenuates thymic cancer cell proliferation and promotes apoptosis via the miR-145-5p/HMGA2 pathway
}

\author{
SHENG TAN $^{1}$ and JILI CHEN ${ }^{2}$ \\ Departments of ${ }^{1}$ Cardiovascular Surgery and ${ }^{2}$ Ophthalmology, Affiliated Hospital of \\ Xuzhou Medical University, Xuzhou, Jiangsu 221000, P.R. China
}

Received May 9, 2020; Accepted February 15, 2021

DOI: $10.3892 / \mathrm{ol} .2021 .12846$

\begin{abstract}
Metastasis-associated-lung-adenocarcinomatranscript-1 (MALAT1) is a long non-coding RNA that is considered a potential tumor marker. The present study aimed to investigate the effect and mechanism of MALAT1 on cell proliferation and apoptosis in thymic cancer cells. IU-TAB-1, A549, HCT-116 and 293T cells were screened by reverse transcription-quantitative PCR to assess high-mobility group AT-hook 2 (HMGA2) expression in various types of cancer cells and were transfected with small interfering (si)RNA targeting MALAT1 (si-MALAT1). Cell proliferation was evaluated by Cell Counting Kit- 8 assay. Cell apoptosis and cell cycle were examined using flow cytometry. The protein expression of cyclin D1, cyclin E, Bax, Bcl-2 and HMGA2 was determined by western blot analysis, while the associations between MALAT1 and microRNA (miR)-145-5p and between HMGA2 and miR-145-5p were determined by luciferase reporter assay. Among the four cell lines evaluated, IU-TAB-1 showed the highest expression of MALAT1; thus, IU-TAB-1 cells were selected for subsequent experiments. Compared with the findings in the control group, si-MALAT1 significantly decreased the cell proliferation of IU-TAB-1 cells, whereas the apoptosis levels and number of cells in $G_{2}$ phase were increased. The protein expression levels of cyclin D1, cyclin E, Bcl-2 and HMGA2 were significantly decreased in the si-MALAT1 group compared with those in the control group, while Bax levels were significantly increased. After treatment with si-MALAT1 in combination with miR-145-5p mimics or inhibitors, cell proliferation and apoptosis were respectively enhanced and inhibited in IU-TAB-1 cells. miR-145-5p inhibited the luciferase activity of IU-TAB-1 cells transfected with the MALAT1 or HMGA2 3' untranslated
\end{abstract}

Correspondence to: Dr Sheng Tan, Department of Cardiovascular Surgery, Affiliated Hospital of Xuzhou Medical University, 99 West Huaihai Road, Xuzhou, Jiangsu 221000, P.R. China E-mail: 15951938580@163.com

Key words: metastasis-associated-lung-adenocarcinoma-transcript-1, proliferation, apoptosis, thymic cancer cell, microRNA-145-5p, high-mobility group AT-hook 2 region. In conclusion, si-MALAT1 significantly attenuated cell proliferation and apoptosis via the miR-145-5p/HMGA2 pathway in thymic cancer cells.

\section{Introduction}

Thymic cancer is a thymic epithelial tumor with strong metastatic characteristics, and 50-65\% of patients exhibit distant metastases when diagnosed (1). At present, surgical resection is the primary method for treating thymic cancer (2), but the application of surgical resection has limitations. Therefore, the identification of novel diagnostic markers and therapeutic targets is of great importance for the clinical treatment of thymic cancer.

Metastasis-associated-lung-adenocarcinoma-transcript-1 (MALAT1) is a long non-coding RNA (lncRNA) that cannot be translated into protein in vitro (3). Previous studies have shown that MALAT1 is closely associated with various diseases, including lung cancer, renal cell carcinoma and esophageal squamous cell carcinoma (4-6). MALAT1 can promote cervical cancer cell proliferation and migration, and can regulate the expression of genes associated with apoptosis (7). Han et al (8) reported that MALAT1 could promote cancer cell proliferation and migration by activating the ERK/MAPK pathway. High expression levels of MALAT1 can be a marker of poor prognosis in patients with hepatocellular carcinoma (9). Therefore, MALAT1 is considered a potential tumor marker.

High-mobility group AT-hook 2 (HMGA2) belongs to the non-histone chromosome high-mobility group family, and is located on chromosome 12q 13-15 (10). The human HMGA2 gene consists of five exons and four introns (10). HMGA2 functions as a transcription factor by altering the structure of chromatin, and by interacting with DNA and target proteins (10). HMGA2 is usually highly expressed in numerous malignant tumors and is closely associated with increased invasiveness (11-15). Tan et al (unpublished data) showed that small interfering (si) RNA targeting HMGA2 attenuated epithelial-mesenchymal transition (EMT) in thymic cancer cells via the Wnt/ $\beta$-catenin pathway. The present study investigated the effects of inhibiting the expression of MALAT1 on the proliferation and apoptosis of thymic cancer cells. Further molecular experiments were performed to verify whether si-MALAT1 exerted its effect by regulating the expression of 
HMGA2. The present study may provide some insights into the treatment of thymic cancer.

\section{Materials and methods}

Cell culture and transfection. The thymic cancer cell line IU-TAB-1 was obtained from Applied Biological Materials, Inc. (cat. no. T8001), while 293T, A549 and HCT-116 cells (used as controls) were provided by the Stem Cell Bank of the Chinese Academy of Sciences. Cells were cultured in RPMI-1640 medium (293T cells) (cat. no. SH30022.01B; HyClone; Cytiva), Prigrow II medium (IU-TAB-1 cells) (cat. no. TM002; Abmgood), F-12K medium (A549 cells) (cat. no. 21127-022; Gibco; Thermo Fisher Scientific, Inc.) or McCoy's 5A medium (HCT-116 cells) (cat. no. 16600-082; Gibco; Thermo Fisher Scientific, Inc.) supplemented with $10 \%$ fetal bovine serum (cat. no. 10270-106; Gibco; Thermo Fisher Scientific, Inc.) in an atmosphere containing $5 \% \mathrm{CO}_{2}$ and $95 \%$ atmospheric air at $37^{\circ} \mathrm{C}$ for $24 \mathrm{~h}$. The medium was replaced every $24 \mathrm{~h}$ and the cells were subcultured or cryopreserved when the cell density reached $70-80 \%$.

The sequence of the full-length cDNA of MALAT1 (accession no. NR_002819.4) was obtained from the NCBI database. IU-TAB-1 cells were transfected with MALAT1 siRNA using Lipofectamine ${ }^{\circledR} 2000$ reagent (cat. no. 13778030; Invitrogen; Thermo Fisher Scientific, Inc.) according to the manufacturer's instructions. Briefly, 100 pmol siRNA and $5 \mathrm{ml}$ Lipofectamine ${ }^{\circledast}$ RNAiMAX were added to $250 \mu \mathrm{l}$ Opti-MEM at $4^{\circ} \mathrm{C}$ for $20 \mathrm{~min}$, and then the mixture was added to the cell culture plates and incubated at $37^{\circ} \mathrm{C}$ in a $5 \% \mathrm{CO}_{2}$ incubator for $48 \mathrm{~h}$ before subsequent experiments. The cells were divided into five groups: Control (untransfected cells), MALAT1-siRNA1, MALAT1-siRNA2, MALAT1-siRNA3 and non-targeting negative control (NC). The transfection efficiency was determined by RT-qPCR.

Cell Counting Kit-8 (CCK-8) assay. IU-TAB-1 cells were seeded in a 96-well plate at $5 \times 10^{3}$ cells $/ \mathrm{ml}$ using Prigrow II medium containing $10 \%$ fetal bovine serum. Cells from each group were treated for $48 \mathrm{~h}$. To evaluate cell proliferation, $10 \mu$ l CCK-8 solution (cat. no. C1706; Bioswamp Wuhan Bienle Biotechnology Co., Ltd.) was added to each well, and the cells were cultured at $37^{\circ} \mathrm{C}$ for $4 \mathrm{~h}$. The optical density at $450 \mathrm{~nm}$ was measured using a plate reader (Multiskan FC; Thermo Fisher Scientific, Inc.).

Flow cytometry. IU-TAB-1 cells were cultured for $24 \mathrm{~h}$ and then harvested. Next, $1 \mathrm{ml}$ pre-cooled PBS was added before centrifuging the cells at $1,000 \mathrm{xg}$ at $4^{\circ} \mathrm{C}$ for $5 \mathrm{~min}$. Next, $10 \mu \mathrm{l}$ Annexin V-FITC and $10 \mu 1$ propidium iodide were added. The apoptotic rate and cell cycle were detected using flow cytometry (NovoCyte; Agilent Technologies, Inc.), and the data were analyzed with CytExpert software (Beckman Coulter, Inc.; version 2.0). One-step fluorescence compensation strategy was used to eliminate any interference with the fluorescein isothiocyanate channel.

$R T-q P C R$. The expression levels of HMGA2, MALAT1 and miR-145-5p in each cell line were detected by RT-qPCR. Total RNA was extracted from $1 \times 10^{6}$ cells using TRIzol ${ }^{\circledR}$ reagent according to the manufacturer's protocol (Invitrogen; Thermo Fisher Scientific, Inc.). cDNA was synthesized using a reverse transcriptase kit (cat. no. 639505; Takara Bio, Inc.). qPCR was performed with a CFX Connect 96 Real-Time PCR Detection System (Bio-Rad Laboratories, Inc.) using SYBR Green PCR kit (cat. no. KM4101; Kapa Biosystems; Roche Diagnostics). Each reaction was conducted in duplicate using the following thermocycling conditions: Denaturation at $95^{\circ} \mathrm{C}$ for $3 \mathrm{~min}$, followed by 39 cycles of $95^{\circ} \mathrm{C}$ for $5 \mathrm{sec}, 56^{\circ} \mathrm{C}$ for $10 \mathrm{sec}$ and $72^{\circ} \mathrm{C}$ for $25 \mathrm{sec} ; 65^{\circ} \mathrm{C}$ for $5 \mathrm{sec}$ and $95^{\circ} \mathrm{C}$ for $50 \mathrm{sec}$. The results were analyzed by the $2^{-\Delta \Delta \mathrm{Cq}}$ method (16). GAPDH and U6 were used as the reference genes for mRNA and miRNA, respectively. The primers were designed and provided by Wuhan Tianyihuiyuan Biotechnology Co., Ltd., and their sequences are listed in Table I.

Western blotting. After treatment for $48 \mathrm{~h}$, cells were washed with cold PBS and subjected to a lysis buffer (cat. no. 180006; Bioswamp Wuhan Bienle Biotechnology Co., Ltd.), and the proteins were quantified using the bicinchoninic acid assay kit (cat. no. 180007; Bioswamp Wuhan Bienle Biotechnology Co., Ltd.). Proteins ( $20 \mu \mathrm{g} /$ lane) were separated via $12 \%$ SDS-PAGE and were then transferred onto PVDF membranes. The membranes were blocked with a buffer containing 5\% non-fat milk in PBS with $0.05 \%$ Tween-20 for $2 \mathrm{~h}$ at room temperature and then incubated with the following primary antibodies (all from Bioswamp Wuhan Bienle Biotechnology Co., Ltd.): Anti-HMGA2 (1:1,000; cat. no. PAB40807), anti-cyclin D1 $(1: 1,000$; cat. no. MAB37160), anti-cyclin E $(1: 1,000$; cat. no. PAB36461), anti-Bax (1:1,000; cat. no. MAB30681), anti-Bcl-2 (1:1,000; cat. no. PAB30041) and anti-GAPDH (1:2,000; cat. no. PAB36264) overnight at $4^{\circ} \mathrm{C}$. After three washes with PBS with $10 \%$ Tween-20, the membranes were incubated with horseradish peroxidase-conjugated secondary goat anti-rabbit IgG (1:20,000; cat. no. PAB160011; Bioswamp Wuhan Bienle Biotechnology Co., Ltd.) for $2 \mathrm{~h}$ at $4^{\circ} \mathrm{C}$. Protein bands were visualized by enhanced chemiluminescence detection (Tanon-5200; Tanon Science and Technology Co., Ltd.) and analyzed using Tanon GIS software (version 4.2; Tanon Science and Technology Co., Ltd.). GAPDH was used as a loading control for normalization.

Luciferase reporter assay. IU-TAB-1 cells were co-transfected with $50 \mathrm{nM}$ miR-145-5p mimics or miR-145-5p inhibitor plus 200 ng MALAT1-3'-UTR or HMGA2-3'-UTR using Lipofectamine ${ }^{\circledR} 2000$ (Invitrogen; Thermo Fisher Scientific, Inc.) following the manufacturer's instructions. After $48 \mathrm{~h}$, the cells were lysed using the Dual-Luciferase Reporter Assay System (Promega Corporation), and luciferase activity was measured using a GloMax20/20 Luminometer (Promega Corporation). Luciferase activity was normalized to the Renilla luciferase signal in IU-TAB-1 cells. The constructs were prepared as follows: MALAT1-3'-UTR sense strand, 5'-CTA GCTTGGAGAAGATAGAAGTTTGAAGTGGAAAACTGG AAGACAGAAGTACGGGAAGGCGAAGAAAAG-3' and antisense strand, 5'-CTAGACTTTTCTTCGCCTTCCCGT ACTTCTGTCTTCCAGTTTTCCACTTCAAACTTCTATCTTCTCCAA-3; HMGA2-3'-UTR sense strand, 5'-CTAGCAAGACCCAAA GGCAGCAAAAACAAGAGTCCCTCTAAAGCAGCTCAA AAGAAAGCAGAAGCCACTG-3' and antisense strand, 
Table I. Primer sequences.

\begin{tabular}{ll}
\hline Primer & \multicolumn{1}{c}{ Sequence (5'-3') } \\
\hline HMGA2-F & TTCAGCCCAGGGACAA \\
HMGA2-R & CCAGGCAAGGCAACAT \\
MALAT1-F & TAACCAGGCATAACAC \\
MALAT1-R & CGAAGACACAGAGACC \\
miR-145-5p-F & GGGGTCCAGTTTTCCCAG \\
miR-145-5p-R & AACTGGTGTCGTGGAGTCGGC \\
U6-F & CTCGCTTCGGCAGCACA \\
U6-R & AACGCTTCACGAATTTGCGT \\
GAPDH-F & CCACTCCTCCACCTTTG \\
GAPDH-R & CACCACCCTGTTGCTGT
\end{tabular}

MALAT1, metastasis-associated-lung-adenocarcinoma-transcript-1; HMGA2, high-mobility group AT-hook 2; F, forward; R, reverse; miR, microRNA.

\section{5'-CTAGACAGTGGCTTCTGCTTTCTTTTGAGCTGCTTT AGAGGGACTCTTGTATTTTGCTGCCTTTGGGTCTT-3'.}

Statistical analysis. All data are presented as the mean \pm standard deviation. Statistical analysis was performed using one-way analysis of variance followed by Tukey's test for multiple comparisons using SPSS 19.0 software (IBM Corp.). All experiments were performed independently three times. All figures were prepared using GraphPad Prism 5.0 software (GraphPad Software, Inc.). P $<0.05$ was considered to indicate a statistically significant difference.

\section{Results}

HMGA2, MALAT1 and miR-145-5p expression in IU-TAB-1, A549, HCT-116 and $293 T$ cells. 293 T cells are a normal human renal epithelial cell line, which is often used as a control group for detecting tumor cytokine expression. As shown in Fig. 1, compared with 293T cells, mRNA expression of HMGA2 and MALAT1 was significantly increased in IU-TAB-1, A549 and HCT-116 cells $(\mathrm{P}<0.05)$, while the expression of miR-145-5p was significantly decreased $(\mathrm{P}<0.05)$. Among all the cell lines evaluated, IU-TAB-1 cells showed the largest fold change in HMGA2 and MALAT1 expression, as well as a significant change in miR-145-5p expression. Therefore, IU-TAB-1 cells were selected for subsequent experiments.

Inhibition of MALAT1 expression. The mRNA expression levels of MALAT1 in the control, MALAT1-siRNA1, MALAT1-siRNA2, MALAT1-siRNA3 and NC groups confirmed MALAT1 silencing following siRNA transfection (Fig. 2). The results revealed that all three MALAT1 siRNA molecules significantly downregulated the expression of MALAT1 compared with that of control and NC IU-TAB-1 cells $(\mathrm{P}<0.05)$. MALAT1-siRNA2 resulted in the lowest MALAT1 expression levels among the three siRNA reagents used and was thus used to silence MALAT1 in subsequent experiments.

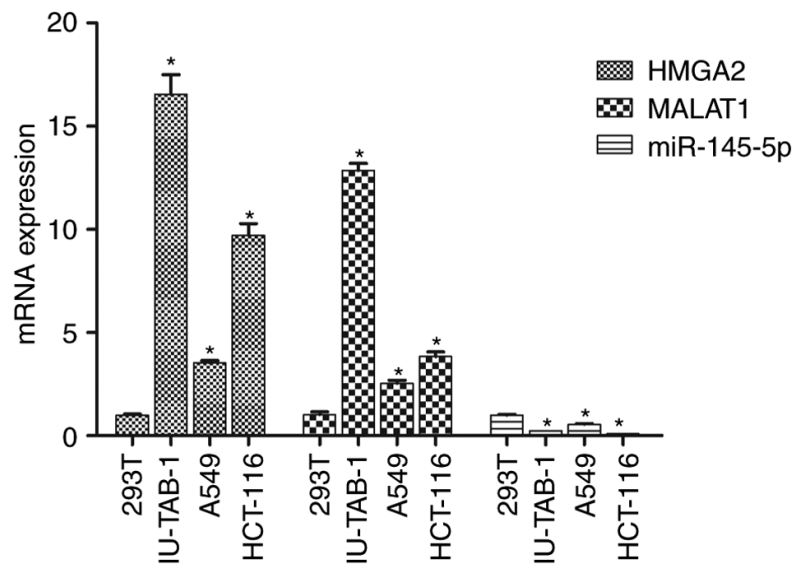

Figure 1. HMGA2, MALAT1 and miR-145-5p expression was determined by reverse transcription-quantitative PCR in A549, HCT-116, IU-TAB-1 and $293 \mathrm{~T}$ cells. Compared with $293 \mathrm{~T}$ cells, mRNA expression of HMGA2 and MALAT1 was significantly increased in IU-TAB-1, A549 and HCT-116 cells, while the expression of miR-145-5p was significantly decreased. ${ }^{*} \mathrm{P}<0.05$ vs. $293 \mathrm{~T}(\mathrm{n}=3)$. HMGA2, high mobility group AT-hook 2; MALAT1, metastasis-associated-lung-adenocarcinoma-transcript-1; miR, microRNA

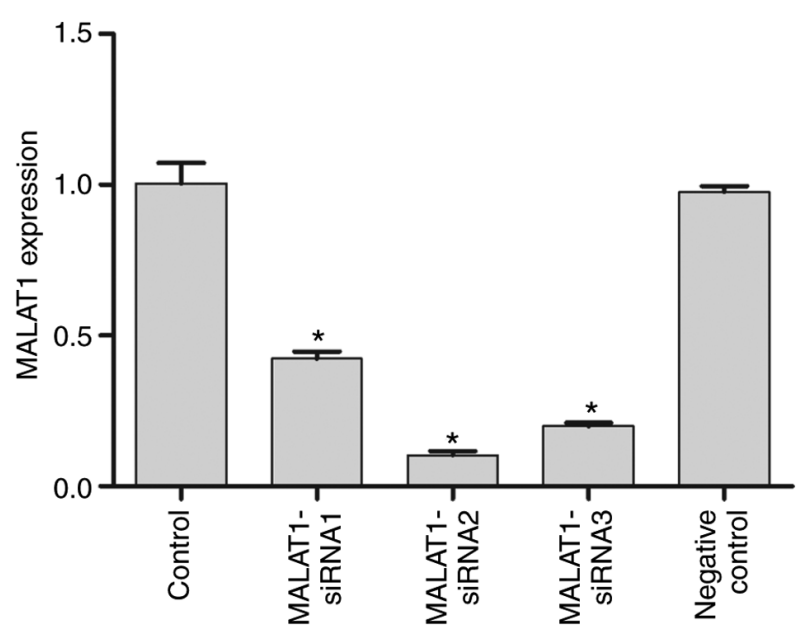

Figure 2. MALAT1 mRNA is repressed in IU-TAB-1 cells. All three MALAT1 siRNA molecules significantly downregulated the expression of MALAT1 compared with the control and NC groups. ${ }^{*} \mathrm{P}<0.05$ vs. control $(n=3)$. MALAT1, metastasis-associated-lung-adenocarcinoma-transcript-1; siRNA, small interfering RNA.

si-MALAT1 attenuates cell proliferation and promotes apoptosis in IU-TAB-1 cells. To examine the effect of MALAT1 on cell proliferation, a CCK-8 assay was performed (Fig. 3A). Transfection with si-MALAT1 significantly suppressed cell proliferation of IU-TAB-1 cells compared with the control and NC $(\mathrm{P}<0.05)$. Flow cytometry revealed that si-HMGA2 significantly increased the apoptotic rate of IU-TAB-1 cells compared with that of control and $\mathrm{NC}$ cells $(\mathrm{P}<0.05 ; \mathrm{Fig} .3 \mathrm{~B})$, and significantly decreased the number of cells in $\mathrm{G}_{1}$ phase while increasing the number of cells in $\mathrm{G}_{2}$ phase $(\mathrm{P}<0.05$; Fig. 3C). Next, the expression of cell cycle and apoptosis-related proteins was examined (Fig. 3D). The protein expression of cyclin D1, cyclin E, Bcl-2 and HMGA2 significantly decreased, whereas that of Bax significantly increased $(\mathrm{P}<0.05$ in all cases) compared with the control and NC groups, indicating that MALAT1 silencing promoted apoptosis and inhibited cell cycle progression in IU-TAB-1 cells . 
A

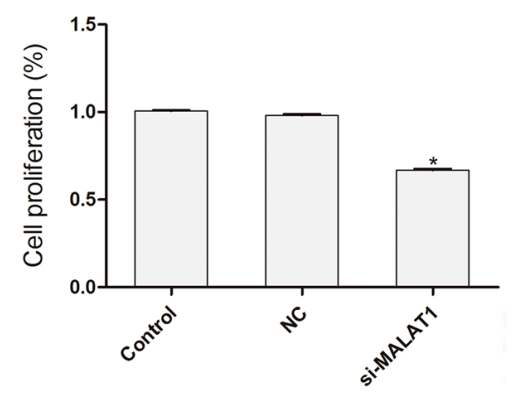

C

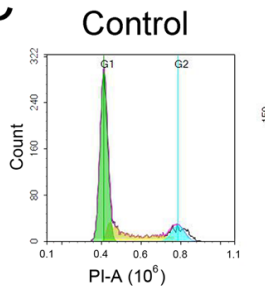

Si-MALAT1

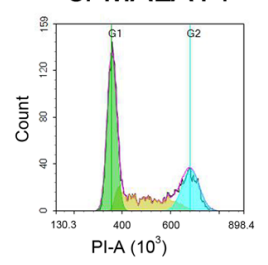

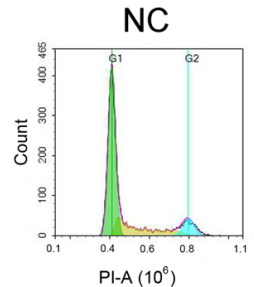

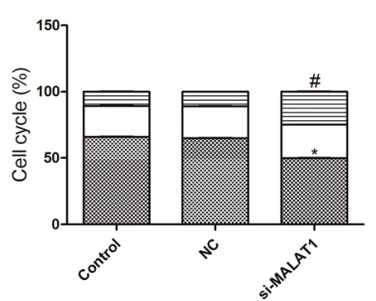

$\mathrm{B}$
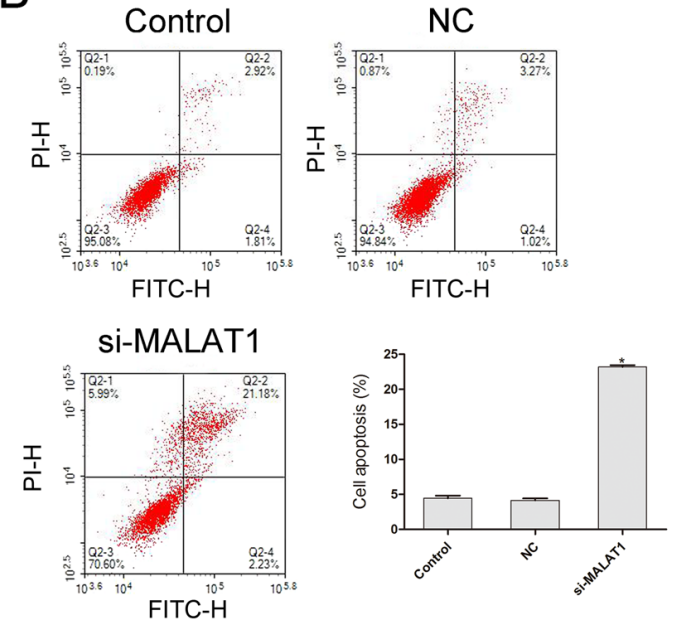

$\mathrm{D}$

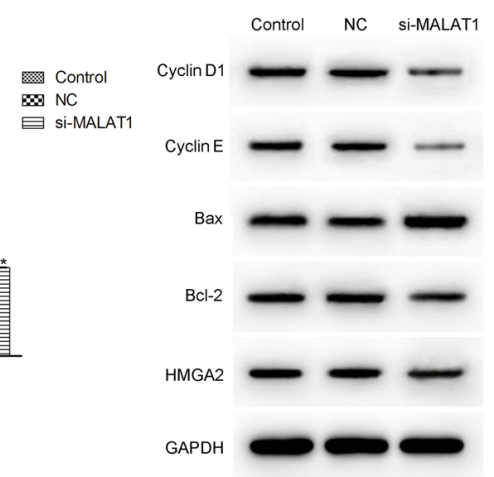

Figure 3. Effect of si-MALAT1 on cell proliferation and apoptosis in IU-TAB-1 cells. (A) si-MALAT1 inhibited the cell proliferation of IU-TAB-1 cells. (B) Cell apoptosis and $(\mathrm{C})$ cell cycle progression were analysed by flow cytometry. ${ }^{*} \mathrm{P}<0.05$ cells in $\mathrm{G}_{1}$ phase vs. Control ( $\mathrm{n}=3$ ); ${ }^{*} \mathrm{P}<0.05$ cells in $\mathrm{G}_{2}$ phase vs. Control $(\mathrm{n}=3)$. (D) Cell cycle and apoptosis-related proteins were detected by western blotting. Compared with the control and NC groups, si-MALAT1 significantly suppressed cell proliferation, the number of cells in $\mathrm{G}_{1}$ phase and the expression of cyclin D1, cyclin E, Bcl-2 and HMGA2, while it increased the apoptotic rate, the number of cells in $\mathrm{G}_{2}$ phase and the expression of $\mathrm{Bax}$. ${ }^{*} \mathrm{P}<0.05$ vs. control $(\mathrm{n}=3)$. MALAT1, metastasis-associated-lung-adenocarcinoma-transcript-1; HMGA2, high mobility group AT-hook 2; NC, negative control; si, small interfering; FITC, flurorescein isothiocyanate; PE, phycoerythrin.

Association between MALAT1 and miR-145-5p, and between HMGA2 and miR-145-5p. The transfection efficiency of miR-145-5p mimics and inhibitor was first examined (Fig. 4A). Compared with that of the control and NC groups, the expression of miR-145-5p in the miR-145-5p mimics group increased significantly $(\mathrm{P}<0.05)$, while it decreased significantly in the miR-145-5p inhibitor group $(\mathrm{P}<0.05)$, suggesting that the transfections of miR-145-5p mimics and inhibitor were successful. To study the association between miR-145-5p and MALAT1, a dual-luciferase reporter assay was conducted. The luciferase reporter vector pmirGLO-MALAT1 3'-UTR was co-transfected with miR-145-5p mimics or inhibitor. The results shown in Fig. 4B indicate that, compared with the control group, the relative luciferase activity of other groups did not change significantly ( $\mathrm{P}>0.05$ ), except for the pmirGO-MALAT1+miR-145-5p mimics group $(\mathrm{P}<0.05)$, suggesting that miR-145-5p specifically binds to MALAT1. Next, the expression of miR-145-5p was evaluated, and the results showed that si-MALAT1 could significantly increase the expression of miR-145-5p $(\mathrm{P}<0.05)$ (Fig. 4C). To study the effect of miR-145-5p on the biological characteristics of IU-TAB-1 cells, miR-145-5p inhibitor and miR-145-5p mimics and si-MALAT1 were used. As shown in Fig. 4D and E, compared with that of the control group, the cell proliferation rate of the miR-145-5p inhibitor group was significantly increased and the apoptosis rate was decreased $(\mathrm{P}<0.05)$, while the proliferation rate of the miR-145-5p mimics group was significantly decreased and the apoptosis rate was significantly increased $(\mathrm{P}<0.05)$. Compared with the control group, the proliferation rate in miR-145-5p mimics + si-MALAT1 group was significantly decreased $(\mathrm{P}<0.05)$, while this group exhibited the highest apoptosis rate (Fig. 4D and E), indicating that there may be a synergistic effect between si-MALAT1 and miR-145-5p mimics. Compared with the control, the number of cells in $\mathrm{G}_{1}$ phase in the miR-145-5p inhibitor group was significantly increased $(\mathrm{P}<0.05)$ and in the miR-145-5p inhibitor + si-MALAT1 group it was significantly decreased $(P<0.05)$, the number of cells in $G_{2}$ phase in the miR-145-5p inhibitor group was significantly decreased $(\mathrm{P}<0.05)$ and in the miR-145-5p inhibitor + si-MALAT1 group it was significantly increased $(\mathrm{P}<0.05)$, while the miR-145-5p mimics and miR-145-5p mimics + si-MALAT1 group had the opposite effects (Fig. 4F). As shown in Fig. 4G, compared with the control, the expression levels of Cyclin D1, Cyclin E, Bcl-2 and HMGA2 in the miR-145-5p inhibitor group were significantly increased $(\mathrm{P}<0.05)$, and in the miR-145-5p inhibitor + si-MALAT1 group they were significantly decreased $(\mathrm{P}<0.05)$, while Bax expression in the miR-145-5p inhibitor group was significantly decreased $(\mathrm{P}<0.05)$ and in the miR-145-5p 
A

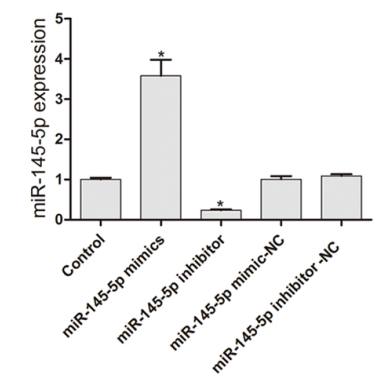

B

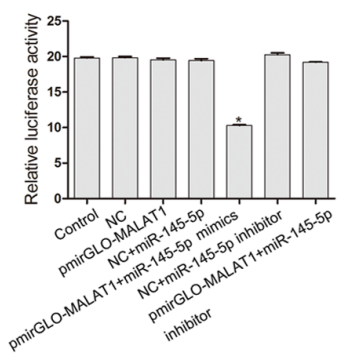

C

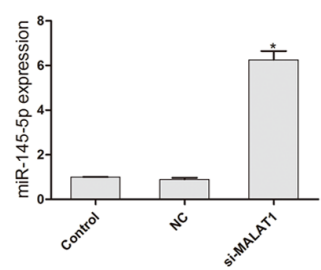

D

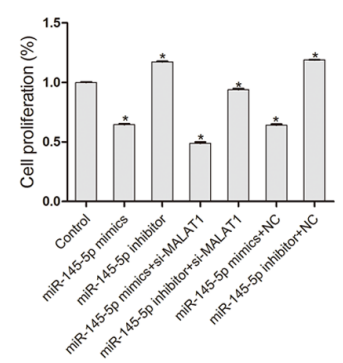

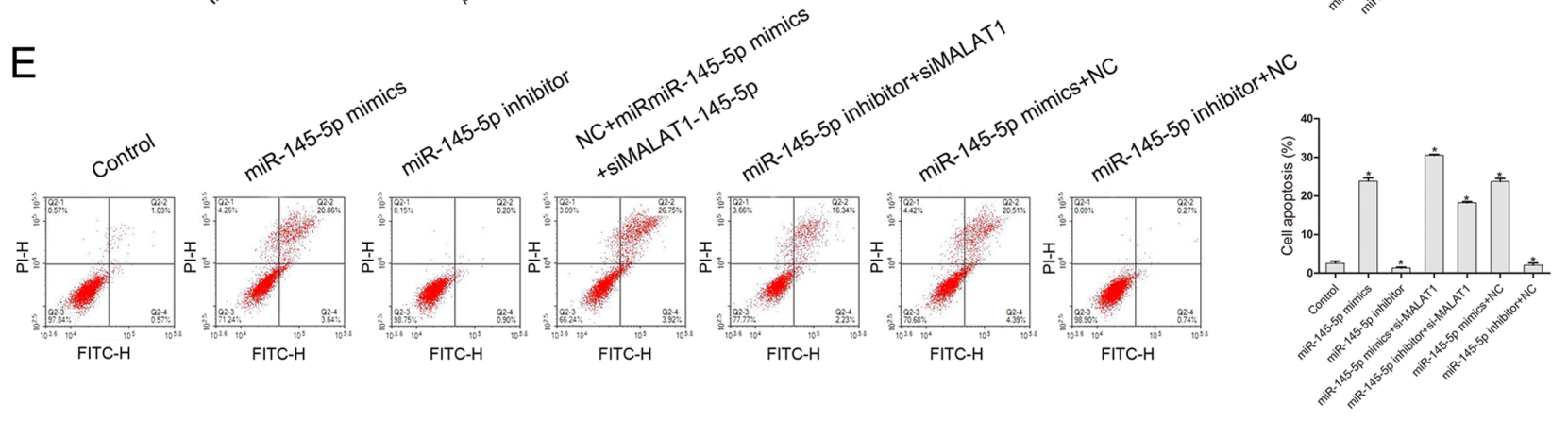

F
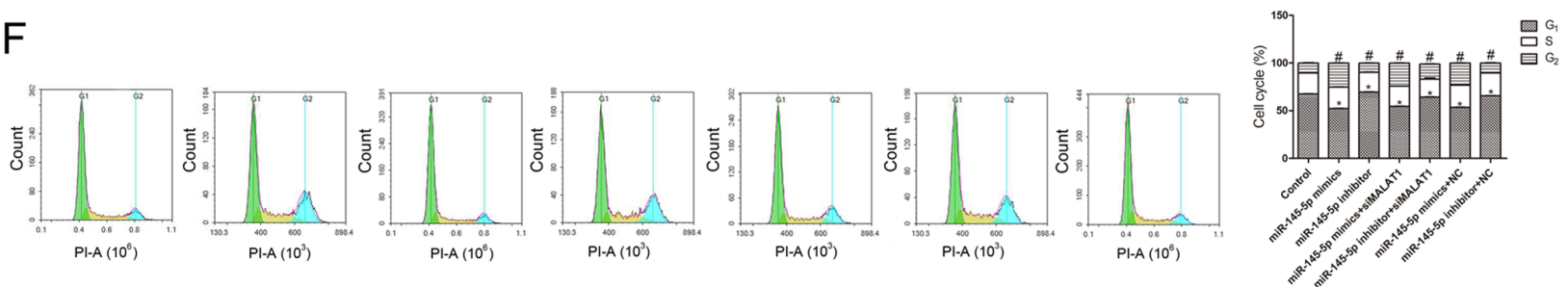

G

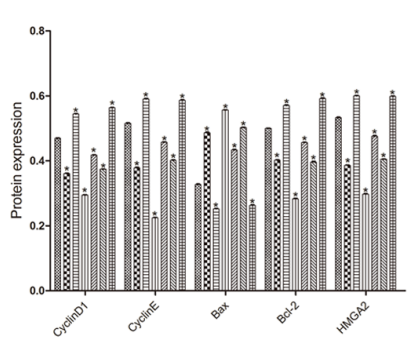

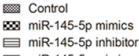

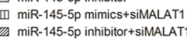

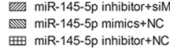

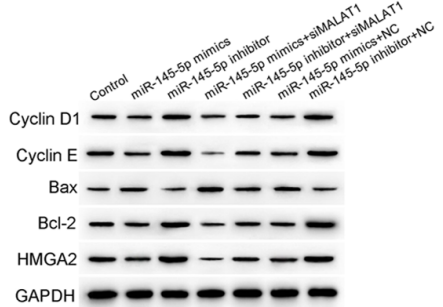

$\mathrm{H}$

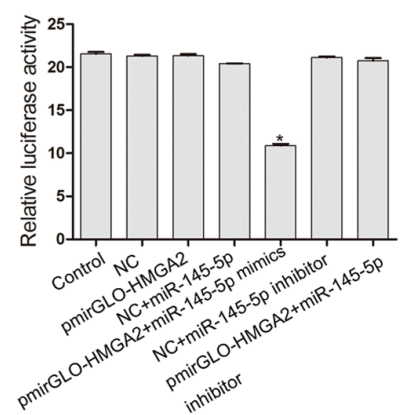

Figure 4. Association between MALAT1 and miR-145-5p, and between HMGA2 and miR-145-5p. (A) Transfection efficiency of miR-145-5p mimics/inhibitors, as evaluated by RT-qPCR. (B) The association between MALAT1 and miR-145-5p was analyzed by luciferase reporter assay. (C) miR-145-5p expression was

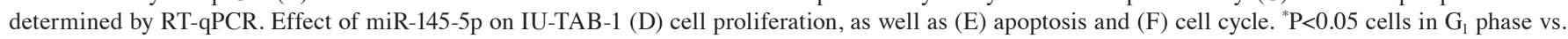
Control ( $\mathrm{n}=3) ;{ }^{\prime} \mathrm{P}<0.05$ cells in $\mathrm{G}_{2}$ phase vs. Control $(\mathrm{n}=3)$. $(\mathrm{G})$ Expression of apoptosis-related proteins were detected by western blotting. (H) Association between HMGA2 and miR-145-5p was detected by luciferase reporter assay. " $\mathrm{P}<0.05$ vs. Control (n=3). MALAT1, metastasis-associated-lung-adenocarcinoma-transcript-1; HMGA2, high mobility group AT-hook 2; NC, negative control; miR, microRNA; RT-qPCR, reverse transcription-quantitative PCR; si, small interfering.

inhibitor + si-MALAT1 group they were significantly increased $(\mathrm{P}<0.05)$. On the other hand, Cyclin D1, Cyclin E, Bcl-2 and HMGA2 expression in the miR-145-5p mimics group and miR-145-5p mimics + si-MALAT1 group was significantly decreased compared with the control, while Bax expression was significantly increased $(\mathrm{P}<0.05$; Fig. $4 \mathrm{G})$. The association between HMGA2 and miR-145-5p was examined. As shown in Fig. 4H, compared with the control group, the relative luciferase activity of other groups did not change significantly $(\mathrm{P}>0.05)$, except for the pmirGLO-HMGA2+miR-145-5p mimics group $(\mathrm{P}<0.05)$, suggesting that there was specific binding between HMGA2 and miR-145-5p.

\section{Discussion}

The occurrence and development of malignant tumors are associated with cell proliferation, apoptosis, invasion and metastasis. Abnormal lncRNA expression can change the 
above biological processes, and plays a key role in tumorigenesis and development (17). MALAT1 is an 8.7-kb gene located on human chromosome 11q13, which is controlled by multiple promoters. It is widely distributed in mammalian normal tissues and abnormally expressed in multiple malignant tumors, affecting tumor proliferation, apoptosis, invasion, metastasis and drug resistance (3). Whether MALAT1 is overexpressed in thymic cancer has not yet been determined. IU-TAB-1, A549 and HCT-116 are common types of tumor cells. Studies (11-15) have shown that MALAT1 is highly expressed in a variety of malignant tumors, such as lung, colon and ovarian cancer, and its enhanced expression is closely associated with enhanced tumor aggressiveness and disease prognosis. Therefore, the present study hypothesized that the occurrence and development of thymic cancer may also be associated with abnormally high MALAT1 expression. The present study detected the expression of MALAT1 in the thymic cancer cell line IU-TAB-1, and it used non-small cell lung cancer A549, human colon cancer HCT-116 and 293T cells as controls. Our results showed that the expression of MALAT1 was the highest in IU-TAB-1 cells, suggesting that MALAT1 is highly expressed in thymic cancer.

MALAT1 acts as a competitive endogenous messenger RNA (18). Liu et al (19) found that MALAT1 can inhibit the expression of the target gene growth factor receptor-bound protein 2 in cervical cancer cells by competitively binding to miR-124, thereby inhibiting the growth and invasion of cervical cancer cells, and increasing their apoptosis. MALAT1 knockout inhibits the migration and invasion of gallbladder cancer cells, and increases their apoptosis (20). Yang et al (21) reported that MALAT1 also has a role in regulating the cell cycle. MALAT1 is expressed at high levels in cell nuclei. At the $\mathrm{G}_{2} / \mathrm{M}$ phase, MALAT1 is localized in the cytoplasm, where interacts with a large number of nuclear factors and heterogeneous nuclear ribonucleoprotein (hnRNP) C protein (22). Downregulating the expression of MALAT1 causes cytoplasmic ectopic hnRNP C expression in $G_{2} / M$ phase, which, in turn, causes $G_{2} / M$ phase arrest (23). To investigate whether MALAT1 has a similar effect on the proliferation and apoptosis of thymic cancer cells, CCK-8 assay and flow cytometry were used to detect cell proliferation and apoptosis rates in the present study. The results showed that inhibiting the expression of MALAT1 can significantly reduce the proliferation of thymic cancer cells and promote their apoptosis. The cell cycle results showed that, after the inhibition of MALAT1, the cell cycle of thymic cancer cells was mainly blocked in the $\mathrm{G}_{2}$ phase, which is consistent with the results observed in other types of tumor, such as pancreatic cancer, liver cancer and squamous cell carcinoma (23-25). To verify this finding, the expression levels of cell cycle- and apoptosis-related proteins were detected, and the results supported the above conclusions, suggesting that si-MALAT1 can inhibit the proliferation of thymic cancer cells and promote their apoptosis.

miRNA is a type of non-coding RNA that is widely involved in cell growth, differentiation, proliferation and apoptosis (26). miR-145-5p, as a tumor suppressor miRNA, is downregulated in colorectal cancer, and inhibits the proliferation, invasion and migration of colorectal cancer cells. miR-145-5p also plays a suppressive role in lung (27), breast (28), cervical (29) and prostate cancer (30). In the present study, the expression of miR-145-5p in thymic cancer cells was significantly lower than that in $293 \mathrm{~T}$ cells. After promoting the expression of miR-145-5p, the proliferation rate of thymic cancer cells was significantly decreased, and the apoptosis rate was significantly increased. Luciferase reporter assay revealed that MALAT1 specifically binds to miR-145-5p, and the miR-145-5p mimics + si-MALAT1 group had the highest apoptosis rate and lowest cell proliferation rate, indicating that there may be a synergistic effect between si-MALAT1 and miR-145-5p mimics. si-MALAT1 plays a role in inhibiting proliferation and promoting apoptosis by enhancing the expression of miR-145-5p. The present study also confirmed that HMGA2 could specifically bind to miR-145-5p, that HMGA2 was highly expressed in IU-TAB-1 cells and that the expression of HMGA2 was significantly reduced after promoting the expression of miR-145-5p. The apoptosis rate in thymic cancer cells was increased, while the cell proliferation rate was decreased, suggesting that miR-145-5p can inhibit the expression of HMGA2. Sun et al (31) determined via microarray analysis that MALAT1 can regulate EMT by regulating Snail, and knocking down MALAT1 can upregulate the expression of E-cadherin and zona occludens-1, and reduce the activity of $\beta$-catenin and vimentin. Xu et al (32) found that the EMT of breast cancer cells was reduced after inhibiting MALAT1 activity. Tan et al (unpublished data) confirmed that inhibition of HMGA2 activity can inhibit the EMT of thymic cancer cells. Therefore, it was hypothesized that si-MALAT1 can inhibit the activity of HMGA2 by promoting the expression of miR-145-5p, thus, inhibiting the EMT of thymic cancer cells. To confirm this hypothesis, further studies are required. The present study did not use clinical tissues, which limits the clinical significance of the study and is one of its limitations. However, the main aim of the present study was to evaluate the effect of MALAT1 on the proliferation and apoptosis of thymic cancer cells. In future studies, the effect of MALAT1 should be further examined using in vivo experiments. Rong et al (33) observed that MALAT1-knockdown repressed NSCLC tumorigenicity by inhibiting cell proliferation and invasion, and promoting apoptosis via regulating miR-515-5p/EEF2. Although the types of tumor cells investigated in the present study were not consistent with the aforementioned study, the current results confirmed those of the study by Rong et al (33). The present study focused on thymic cancer, which is rarer in clinical practice, and the current results may therefore have a high clinical value and may provide a possible molecular-targeted therapy for patients with thymic cancer.

In conclusion, si-MALAT1 inhibits the proliferation and apoptosis of IU-TAB-1 cells, and its mechanism may involve promoting the expression of miR-145-5p and inhibiting that of HMGA2.

\section{Acknowledgements}

Not applicable.

\section{Funding}

No funding was received. 


\section{Availability of data and materials}

The data used to support the findings of the present study are available from the corresponding author upon request.

\section{Authors' contributions}

ST and JC collected and analyzed the data, and confirm the authenticity of all the raw data. ST drafted the manuscript. JC revised the manuscript. Both authors read and approved the final manuscript.

\section{Ethics approval and consent to participate}

Not applicable.

\section{Patient consent for publication}

Not applicable.

\section{Competing interests}

The authors declare that they have no competing interests.

\section{References}

1. Fink C, Henzler T, Shirinova A, Apfaltrer P and Wasser K Thoracic magnetic resonance imaging: Pulmonary thromboembolism. J Thorac Imaging 28: 171-177, 2013.

2. Qureshi S, West M and Kirk AJB: Surgical resection for thymic malignancy. Lung Cancer 63 (Suppl): S38, 2009.

3. Gutschner T, Monika H and Diederichs S: MALAT1-a paradigm for long noncoding RNA function in cancer. J Mol Med (Berl) 91: 791-801, 2013.

4. Gutschner T, Hämmerle M, Eissmann M, Hsu J, Kim Y, Hung G, Revenko A, Arun G, Stentrup M, Gross M, et al: The Noncoding RNA MALAT1 is a critical regulator of the metastasis phenotype of lung cancer cells. Cancer Res 73: 1180-1189, 2013.

5. Hirata H, Hinoda Y, Shahryari V, Deng G, Nakajima K, Tabatabai ZL, Ishii $\mathrm{N}$ and Dahiya R: Long noncoding RNA MALAT1 promotes aggressive renal cell carcinoma through Ezh2 and interacts with miR-205. Cancer Res 75: 1322-1331, 2015.

6. Hu L, Wu Y, Tan D, Meng H, Wang K, Bai Y and Yang K: Up-regulation of long noncoding RNA MALAT1 contributes to proliferation and metastasis in esophageal squamous cell carcinoma. J Exp Clin Cancer Res 34: 7, 2015.

7. Zhang Y, Wang T, Huang HQ, Li W, Cheng XL and Yang J: Human MALAT-1 long non-coding RNA is overexpressed in cervical cancer metastasis and promotes cell proliferation, invasion and migration. J BUON 20: 1497-1503, 2016.

8. Han Y, Wu Z, Wu T, Huang Y, Cheng Z, Li X, Sun T, Xie X, Zhou Y and Du Z: Tumor-suppressive function of long noncoding RNA MALAT1 in glioma cells by downregulation of MMP2 and inactivation of ERK/MAPK signaling. Cell Death Dis 7: e2123, 2016.

9. Konishi H, Ichikawa D, Yamamoto Y, Arita T, Shoda K, Hiramoto H, Hamada J, Itoh H, Fujita Y, Komatsu S, et al: Plasma level of metastasis-associated lung adenocarcinoma transcript 1 is associated with liver damage and predicts development of hepatocellular carcinoma. Cancer Sci 107: 149-154, 2016

10. Narita M, Narita M, Krizhanovsky V, Nuñez S, Chicas A, Hearn SA, Myers MP and Lowe SW: A novel role for high-mobility group a proteins in cellular senescence and heterochromatin formation. Cell 126: 503-514, 2006.

11. Di Cello F, Hillion J, Hristov A, Wood LJ, Mukherjee M, Schuldenfrei A, Kowalski J, Bhattacharya R, Ashfaq R and Resar LM: HMGA2 participates in transformation in human lung cancer. Mol Cancer Res 6: 743-750, 2008

12. Malek A, Bakhidze E, Noske A, Sers C, Aigner A, Schäfer R and Tchernitsa O: HMGA2 gene is a promising target for ovarian cancer silencing therapy. Int J Cancer 123: 348-356, 2008.
13. Huang ML, Chen CC and Chang LC: Gene expressions of HMGI-C and HMGI(Y) are associated with stage and metastasis in colorectal cancer. Int J Colorectal Dis 24: 1281-1286, 2009.

14. Hristov AC, Cope L, Reyes MD, Singh M, Iacobuzio-Donahue C, Maitra A and Resar LM: HMGA2 protein expression correlates with lymph node metastasis and increased tumor grade in pancreatic ductal adenocarcinoma. Mod Pathol 22: 43-49, 2009.

15. Bartuma H, Panagopoulos I, Collin A, Trombetta D Domanski HA, Mandahl N and Mertens F: Expression levels of HMGA2 in adipocytic tumors correlate with morphologic and cytogenetic subgroups. Mol Cancer 8: 36, 2009.

16. Livak KJ and Schmittgen TDL: Analysis of relative gene expression data using real-time quantitative PCR and the 2(-Delta Delta C(T)) method. Methods 25: 402-408, 2001.

17. Shi D, Zhang Y, Lu R and Zhang Y: The long non-coding RNA MALAT1 interacted with miR-218 modulates choriocarcinoma growth by targeting Fbxw8. Biomed Pharmacother 97: 543-550, 2017.

18. Bai RM and Wang CX: Progress of long non-coding RNA MALAT1 in tumor research. J Clin Oncol 21: 1139-1145, 2016.

19. Liu S, Song L, Zeng S and Zhang L: MALAT1-miR-124-RBG2 axis is involved in growth and invasion of HR-HPV-positive cervical cancer cells. Tumour Biol 37: 633-640, 2016.

20. Wang SH, Zhang WJ, Wu XC, Zhang MD, Weng MZ, Zhou D, Wang JD and Quan ZW: Long non-coding RNA Malat1 promotes gallbladder cancer development by acting as a molecular sponge to regulate miR-206. Oncotarget 7: 37857-37867, 2016.

21. Yang F, Yi F, Han X, Du Q and Liang Z: MALAT-1 interacts with hnRNP C in cell cycle regulation. FEBS Lett 587: 3175-3181, 2013.

22. Miyagawa R, Tano K, Mizuno R, Nakamura Y, Ijiri K, Rakwal R, Shibato J, Masuo Y, Mayeda A, Hirose T and Akimitsu N: Identification of cis- and trans-acting factors involved in the localization of MALAT-1 noncoding RNA to nuclear speckles. RNA 18: 738-751, 2012.

23. Zhang YJ, Tang XM, Shi MM, Wen CL and Shen BY: MiR-216a decreases MALAT1 expression, induces G2/M arrest and apoptosis in pancreatic cancer cells. Biochem Biophys Res Commun 483: 816-822, 2017.

24. Yao K, Zhang GJ, Huang ZM and Yao GX: Expression and function of long non-coding RNA-MALAT1 in liver cancer. Chin J Gen Surg 1: 90-96, 2016.

25. Kangboonruang K, Wongtrakoongate $\mathrm{P}$ and Lertsuwan $\mathrm{K}$, Khachonkham S, Changkaew P, Tangboonduangjit P, Siripoon T, Ngamphaiboon $\mathrm{N}$ and Chairoungdua A: MALAT1 decreases the sensitivity of head and neck squamous cell carcinoma cells to radiation and cisplatin. Anticancer Res 40: 2645-2655, 2020.

26. Esquela-Kerscher A and Slack FJ: Oncomirs-microRNAs with a role in cancer. Nat Rev Cancer 6: 259-269, 2006.

27. Li Y, Li Y, Liu J, Fan Y, Li X, Dong M, Liu H and Chen J: Expression levels of microRNA-145 and microRNA-10b are associated with metastasis in non-small cell lung cancer. Cancer Biol Ther 17: 272-279, 2016.

28. Ding Y, Zhang C, Zhang J, Zhang N, Li T, Fang J, Zhang Y, Zuo F, Tao Z, Tang S, et al: miR-145 inhibits proliferation and migration of breast cancer cells by directly or indirectly regulating TGF- $\beta 1$ expression. Int J Oncol 50: 1701-1710, 2017.

29. Zhou X, Yue Y, Wang R, Gong B and Duan Z: MicroRNA-145 inhibits tumorigenesis and invasion of cervical cancer stem cells. Int J Oncol 50: 853-862, 2017.

30. Xie SG, Xie YY, Zhang LL and Huang Q: Effect of miR-145 targeting DAB2 on the migration and invasion of prostate cancer PC 3 cells. Heredity 36: 50-57, 2014.

31. Sun R, Qin C, Jiang B, Fang S, Pan X, Peng L, Liu Z, Li W, Li Y and Li G: Down-regulation of MALAT1 inhibits cervical cancer cell invasion and metastasis by inhibition of epithelial-mesenchymal transition. Mol Biosyst 12: 952-962, 2016.

32. Xu S, Sui S, Zhang J, Bai N, Shi Q, Zhang G, Gao S, You Z, Zhan C, Liu F and Pang D: Downregulation of long noncoding RNA MALAT1 induces epithelial-to-mesenchymal transition via the PI3K-AKT pathway in breast cancer. Int J Clin Exp Pathol 8: 4881-4891, 2015.

33. Rong F, Liu L, Zou C, Zeng J and Xu Y: MALAT1 promotes cell tumorigenicity through regulating miR-515-5p/EEF2 axis in non-small cell lung cancer. Cancer Manag Res 12: 7691-7701, 2020 .

This work is licensed under a Creative Commons Attribution-NonCommercial-NoDerivatives 4.0 International (CC BY-NC-ND 4.0) License. 